\title{
Dawn-dusk asymmetries and sub-Alfvénic flow in the high and low latitude magnetosheath
}

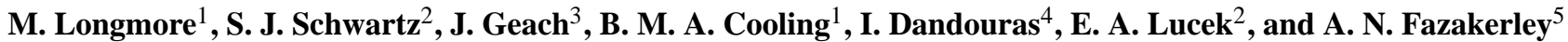 \\ ${ }^{1}$ Astronomy Unit, Queen Mary, University of London, Mile End Road, London, E1 4NS, UK \\ ${ }^{2}$ Blackett Laboratory, Imperial College London, Prince Consort Road, London, SW7 2BW, UK \\ ${ }^{3}$ Department of Physics, University of Durham, South Road, Durham, DH1 3LE, UK \\ ${ }^{4}$ CESR-CNRS, 31028 Toulouse Cedex 4, France \\ ${ }^{5}$ Mullard Space Science Laboratory, Univ. College London, Holmbury St. Mary, Dorking, Surrey, RH5 6NT, London, UK
}

Received: 22 February 2005 - Revised: 11 August 2005 - Accepted: 9 September 2005 - Published: 30 November 2005

\begin{abstract}
We present the results of a statistical survey of the magnetosheath using four years of Cluster orbital coverage. Moments of the plasma distribution obtained from the electron and ion instruments together with magnetic field data are used to characterise the flow and density in the magnetosheath. We note two important differences between our survey and the gasdynamic model predictions: a deceleration of the flow at higher latitudes close to the magnetopause, resulting in sub-Alfvénic flow near the cusp, and a dawn-dusk asymmetry with higher velocity magnitudes and lower densities measured on the dusk side of the magnetosheath in the Northern Hemisphere. The latter observation is in agreement with studies carried out by Paularena et al. (2001), Němeček et al. (2000), and Šafránková et al. (2004). In additon to this we observe a reverse of this asymmetry for the Southern Hemisphere. High-latitude sub-Alfvénic flow is thought to be a necessary condition for steady state reconnection poleward of the cusp.
\end{abstract}

\section{Introduction}

The magnetosheath, a region bounded at its outer edge by the bow shock and at its inner edge by the magnetopause, is an essential element in the solar wind - magnetospheric interaction. The importance of the magnetosheath lies in the fact that it is the medium through which energy and momentum are transported from the solar wind into the Earth's magnetosphere.

In the MHD description the magnetosheath serves to reconfigure the upstream solar wind flow and its frozen-in magnetic field to the state specified by the magnetopause bound-

Correspondence to: M. Longmore

(m.longmore@qmul.ac.uk) ary conditions. This is however a simplification of the reality, since it does not account for kinetic processes operating on small scale lengths which will collectively also contribute to the plasma behaviour. In addition, the appearance and location of the magnetosheath boundaries at any time will depend on the upstream solar wind conditions.

Current estimates of the global plasma properties in the magnetosheath are largely based on the results of the gasdynamic model predictions of Spreiter and Stahara (1980). The Spreiter and Stahara model (Spreiter et al., 1966; Spreiter and Stahara, 1980) assumes that bulk flow properties of the solar wind past a planetary obstacle can be described by the continuum equations of hydrodynamics for a single-component gas (of zero viscosity and thermal conductivity). A simplified non-self-consistent prescription for the magnetic field, which is frozen kinematically to the flow, means magnetic forces are omitted from the momentum equation. Hence some of the observed physics in the Earth's magnetosheath, where the magnetic field is influential, are not described by the model. The model makes the addtional assumption of cylindrical symmetry about the incident flow direction.

Significant effort to consolidate the Spreiter and Stahara model and understand the influence of the magnetic field in the magnetosheath is made in the plasma depletion model proposed by Zwan and Wolf (1976). The model incorporates the effects of the magnetic field on magnetosheath flow. The model follows a magnetic flux tube moving from the bow shock to the magnetopause whilst satisfying the conservation laws along the flux tube. Both diversion of the flow at the bow shock along the magnetic field direction and a "squeezing" effect close to the magnetopause where flux tubes pile up are found to lead to a density depletion at the magnetopause. The result is a net density decrease in regions where the deceleration of the flow is not efficient and diversion of the flow dominates. 
Southwood and Kivelson (1995) later identified a paradox in the formulation of the Zwan and Wolf model, namely that diversion of the flow results in a lower density along the stagnation streamline (where the flow velocity is close to zero) making it difficult to achieve the pressure gradient force required for the plasma to move along the field and for the flux tube pile up and depletion at the magnetopause. This was resolved by proposing the addition of a slow mode compressional front at the end of a diversion dominant region in order to provide the higher thermal pressure required for depletion at the magnetopause boundary.

Another limitation of the Spreiter and Stahara model is that it does not incorporate reconnection processes at the magnetopause. This has been consolidated by the work of Cowley and Owen (1989) and Cooling et al. (2001) who develop the notion of magnetic flux tubes to model the consequences of reconnection at the magnetopause boundary.

Farrugia et al. (1998) compare results from their steadystate MHD model with an inbound crossing of the magnetosheath by AMPTE/IRM close to noon and the ecliptic plane. They reported observation of a depletion layer and an enhanced tangential component flow close to the magnetopause. Similarly Song et al. (1992a,b) have carried out comparisons of observations and MHD models. Fuselier et al. (2002) compared observations of magnetosheath plasma in the high-altitude cusp with gas dynamic and MHD model predictions. They found that gasdynamic models over-estimate the flow velocity adjacent to the magnetopause at high latitudes whilst MHD models which include the effects of magnetic reconnection predicted lower flow velocities than those observed in the same region. A limitation of these comparisons is that they are either based on a single case study or small number of observations.

Statistical studies based on several years of observations in the flanks of the magnetosheath, both in the night and dayside magnetosheath, have been carried out using the Interball-1 data by Šafránková et al. (2004) and Němeček et al. (2000, 2002a,b). The authors concluded that the gasdynamic models were limited in their ability to characterise the flow, density and magnetic field in the magnetosheath, that certain conditions favoured the creation of a depletion layer close to the magnetopause, and that a dawn-dusk asymmetry existed for the ion flux in the magnetosheath which appeared to have some dependence on the $B_{z}$ component of the magnetic field. Similarly Paularena et al. (2001) conducted a survey of the nightside magnetosheath using data from IMP 8 at $-15<X_{G S E}<-20 R_{E}$ and solar wind data from ISEE 1, ISEE 3 and WIND, to investigate magnetosheath structure. The survey revealed a dawn-dusk asymmetry for an era close to solar maximum with higher densities measured on the dawn-side. The Paulerena survey could find no dependence of this asymmetry on the upstream field direction and concluded that some other explanation was required to explain its existence.

More recently Cooling (2003) conducted a survey of the magnetosheath using Geotail data with WIND as an upstream solar wind monitor. In this case the author observed a slight asymmetry in dawn-dusk values of density and velocity. The velocity was found to be lower than that predicted by the Spreiter and Stahara model particularly for locations further downstream of sub-solar point, close to the magnetopause.

The portion of the dayside magnetosheath at high latitudes has not been systematically studied to date. Our aim is to survey this region using four years of Cluster orbits in the dayside magnetosheath in conjunction with upstream solar wind values derived from the ACE spacecraft. The intention is to provide information on the plasma characteristics of the magnetosheath in this region and understand the factors which may be controlling them in a manner which is complimentary to established models and existing research of the magnetosheath region.

\section{Method}

\subsection{Data used}

PEACE (Plasma Electron and Current Experiment) (Johnstone et al., 1997) and CIS (Cluster Ion Spectroscopy) (Rème et al., 2001) 4-s resolution moment data, from the Cluster mission (Escoubet et al., 1997) are used to characterise the bulk parameters in the magnetosheath. In addition, the Cluster prime parameter magnetic field data, (Balogh et al., 2001) at 4-s resolution are used to survey the magnetic field.

ACE SWEPAM and MAG data products provide complimentary data on the upstream solar wind conditions (data was provided by the ACE Science Center). SWEPAM provides 64-s resolution measurements of the ion velocity whilst MAG provides 16-s resolution of the magnetic field. ACE data was available for the duration of the survey (January 2001 until 4 May 2004 ) from both instruments.

\subsection{Plasma moments}

The low order moments of the electron and ion velocity distributions describe the bulk density and velocity characteristics of the magnetosheath. Here PEACE moments are used, which have been corrected for the effects of spacecraft potential and finite range of integration (Geach et al., 2004; Génot and Schwartz, 2004). Typically, the moments are derived from electrons measured in the range $10 \mathrm{eV}-26 \mathrm{keV}$ after removing any contributions from a contaminating photoelectron population. Where the PEACE instrument was powered off in the magnetosheath for a substantial part of the 2003, we have used the CIS HIA (Hot Ion analyser) moments in place of the corrected PEACE moments. These are derived from ions in the $5 \mathrm{eV} / \mathrm{q}-32 \mathrm{keV} / \mathrm{q}$ range.

\subsection{Survey coverage}

Figure 1 shows the total orbital coverage of the magnetosheath from January 2001 to May 2004. Only parts of the orbit lying within the magnetosheath are shown. It is clear 


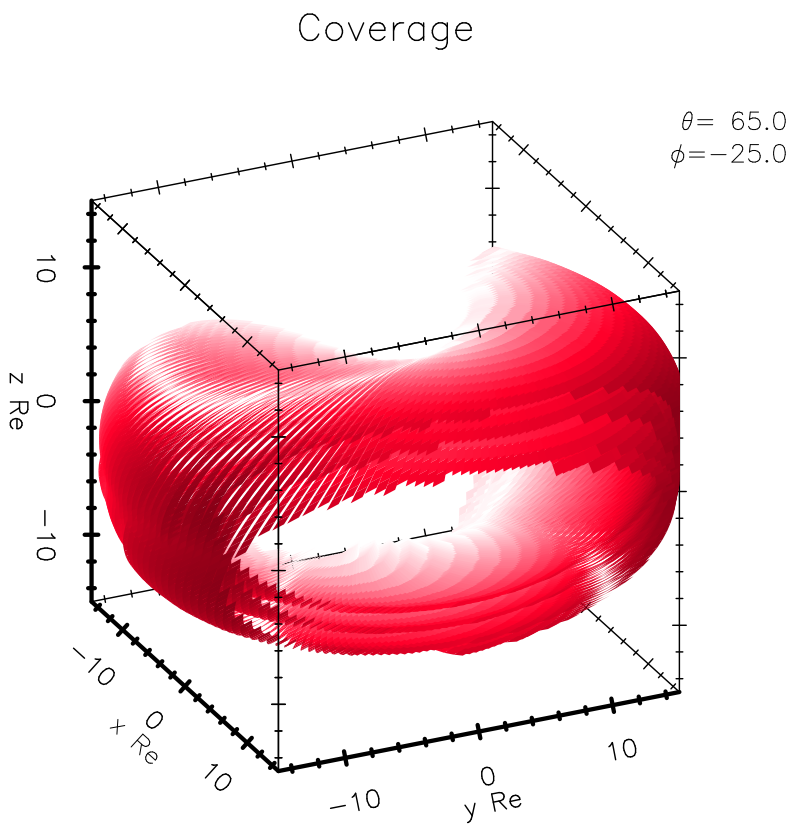

Fig. 1. Cluster orbital coverage of the magnetosheath from January 2001 to May 2004. The spacecraft position from the magnetopause are indicated in red-white shading. Parts of the orbital trajectory lying closest to the magnetopause are indicated in white; those lying further out are indicated in red.

that there is an orbital bias in our survey imposed by the inclined orbit of the Cluster spacecraft; high latitude measurements lie close to the magnetopause whilst conversely low latitude measurements lie close to the bowshock. We note also the poor coverage of the sub-solar region which limits our ability to draw any conclusions about the plasma flow there.

\subsection{Selection of magnetosheath data}

Magnetosheath data were selected by examining each crossing during the survey period. We include an example of a magnetosheath crossing in Fig. 2 to illustrate how the selection is carried out.

A magnetopause boundary and bowshock boundary (thick black lines) are selected from each magnetosheath crossing and all data points obtained during a crossing are normalised to lie between these two points. The bow shock boundary is identified by eye as the sharp jump of $\approx 4$ times the solar wind velocity magnitude, density and magnetic field magnitudes predicted by the Rankine-Hugoniot conservation laws for a shock discontinuity. There is also strong parallel heating of the ions and sharp deceleration of the $V_{x}$ component as it is slowed and deflected around the bow shock boundary. An exact location for the magnetopause boundary is generally more difficult to define. The crossing here corresponds to the high magnetic shear cases of Paschmann et al. (1986) and Phan et al. (1994). In this case the magnetopause crossing is characterised by a field rotation region where the magnetic field increases and the density decreases to the values

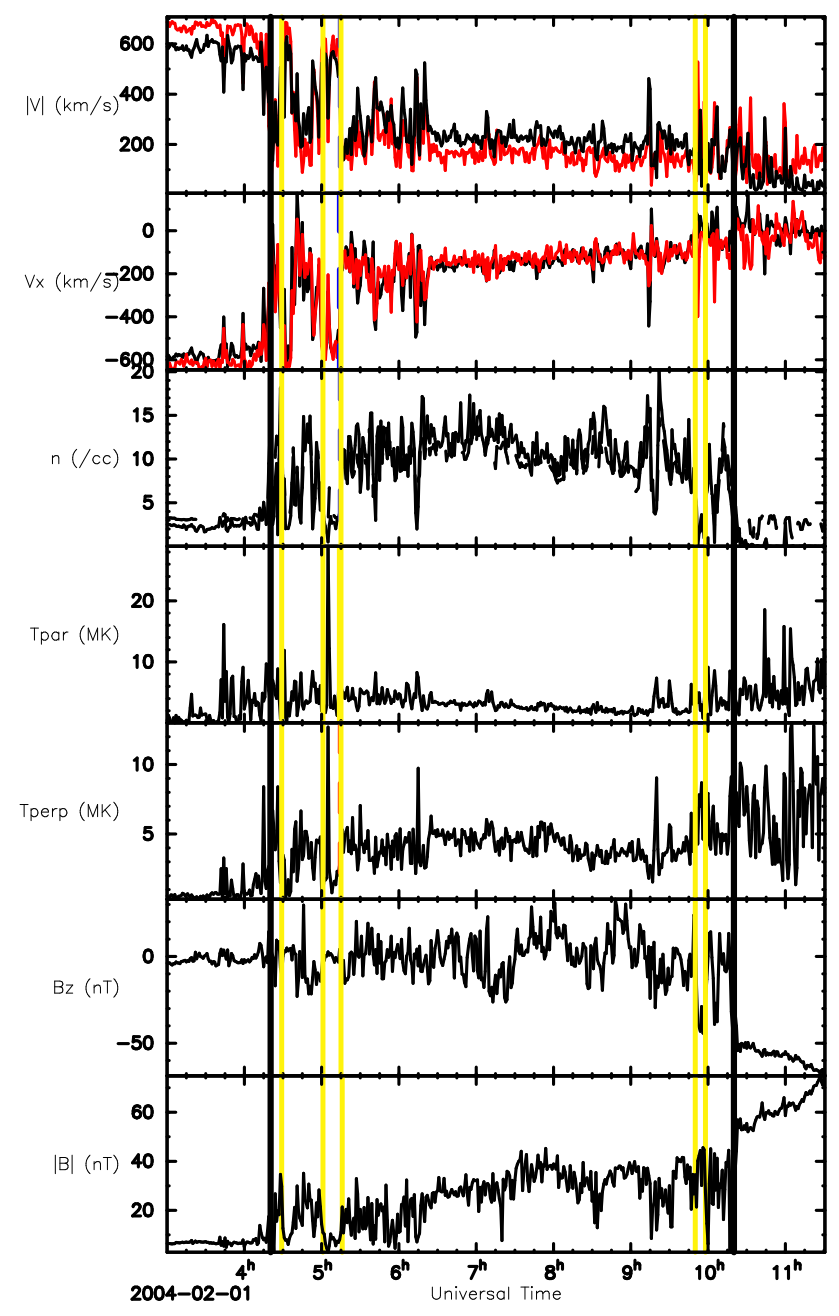

Fig. 2. Selecting the magnetosheath crossing: Electron (red), ion and magnetic field (black) data for a magnetosheath crossing on the February 1st 2004. The spacecraft first encounters the magnetosheath at approximately 4:18 UT, (thick blue line). There are two subsequent re-entries of the spacecraft into the solar wind environment after this time, during the periods 04:25-04:40, 04:5305:14 UT, and an entry by the spacecraft into the magnetosphere between 09:50-09:58. These intervals are indicated by the dashed blue lines and are excluded from our magnetosheath data selection.

expected for magnetospheric plasma. However in the case of low magnetic shear, rotation of the field is often small or absent and a sharp boundary for the magnetopause crossing is more difficult to define. Paschmann et al. (1993) identify a key time for the transition from magnetosheath to magnetospheric plasma in these cases, in which the electron and proton temperatures show discontinuous jumps, and for which a change in the plasma flow direction occurs. On the magnetosheath side of this transition Paschmann et al. (1993) have also identified a layer of density depletion and magnetic field pile-up. In our case we have selected the magnetopause crossing to lie a at the end of the depletion region at the last point before the transition into a magnetospheric plasma regime. 
Figure 2 shows a plot of the behaviour of the various plasma parameters during a typical magnetosheath crossing on the 4 of February 2002. Examining the plasma behaviour in Fig. 2 for the spacecraft transit from the solar wind environment to the magnetosphere we notice gross changes in the overall plasma behaviour and boundaries between 'solar wind' , "magnetosheath" and "magnetospheric" type plasma. The spacecraft first encounter the magnetosheath at approximately 4:18 UT, (thick black line). This is treated as the position of the outer bow shock boundary for normalisation. There are two subsequent re-entries of the spacecraft into the solar wind environment after this time, during the periods 04:25-04:40, 04:53-05:14, and an entry by the spacecraft into the magnetosphere between 09:50-09:58. In Fig. 2 these intervals are indicated by yellow lines and are excluded from our magnetosheath data selection. In this way we attempt to minimise contamination of the database from solar wind and magnetospheric data. The final magnetopause boundary crossing occurs at 10:19 UT and defines the inner boundary for the normalisation.

2.5 Normalisation of magnetosheath data to a model magnetosheath

In order to create a stationary model of the magnetosheath i.e. one in which the boundary positions do not vary with upstream conditions, it is necessary to normalise each measurement within the magnetosheath crossing to a point between the locally measured magnetopause and bowshock. The bowshock and magnetopause positions are selected as described above for an inbound/outbound crossing at the start/end of each crossing. In order to transform to a model frame in which we can can locate each measurement as a function of a geocentric radial, latitude, and phi $(r, \lambda, \phi)$ coordinate it is necessary to firstly scale model boundaries to pass through the selected magnetopause and bowshock locations. For this purpose the Peredo et al. (1995) and Roelof and Sibeck (1993) models are used for the bow shock and magnetpause respectively. Since we scale these models to actual boundary crossing locations, our results are not sensitive to the specific choice of model, although other models, e.g. Shue et al. (2000) may be better suited to predicting the location of the magnetopause for given solar wind parameters.

The distance modulus is then calculated from the spacecraft location along the radial direction to the newly scaled magnetopause and bowshock boundaries and each measurement is normalised to lie within the normalised range 0.0 (magnetopause) to 1.0 (bowshock). In this way it is possible to compare data from different regions of the magnetosheath, obtained during different crossings under varying upstream solar wind conditions.
2.6 Normalisation of data to upstream solar wind conditions

The values of the plasma parameters measured in the magnetosheath depend on the upstream solar wind conditions. For this reason we use ACE at L1 as a monitor of the upstream solar wind conditions. We then normalise the magnetosheath density, velocity and magnetic field measurements to the deduced instantaneous solar wind measurements. We have chosen not to normalise our data to predictions of, say, a gasdynamic model (e.g. Spreiter and Stahara (1980)) in order to minimize the model-dependency of our main results. Additionally, most of our data are drawn from periods of relatively high solar wind Mach number. The dependence of magnetosheath compression ratios with Mach number will therefore have little influence on the results presented here.

Firstly, we show that there are no inaccuracies issues arising from inter-calibration of the ACE and Cluster data sets. We compare solar wind data from both instruments in Fig. 3 and find that the magnetic field and plasma parameters measured at both spacecraft are in excellent agreement.

The instantaneous time lag is then deduced as follows: For Cluster measurements at time $t_{C}$ in the magnetosheath, we find the corresponding value at ACE lagged by the propagation time $\Delta t$, i.e. at a time

$t_{A}=t_{C}-\Delta t$

Since the propagation time through the magnetosheath is small compared to the solar wind transit time from ACE at L1, we take

$\Delta t=|\Delta r| / V_{s w}\left(t_{A}\right)=|\Delta r| / V_{s w}\left(t_{C}-\Delta t\right)$

where $|\Delta r|$ is the distance between ACE and Cluster, and $V_{s w}(t)$ is the solar wind speed measured by ACE at time t. Although this may not be accurate close to the stagnation streamline it is in general a reasonable approximation for the magnetosheath.

For each Cluster observation time $t_{C}$, we solve Eq. (2) iteratively for $\Delta t$ within a $1 \mathrm{hr}$ window. We then normalise the magnetosheath parameters by the ACE measurements at the lagged time.

In order to asses the accuracy of the time lag calculated from Eq. (2), we compare the value of this time lag with the time lag deduced from cross-correlation analysis. The analysis is performed on the ACE and Cluster measurements of $B_{z}$ (GSE). Figure 4 shows ACE and Cluster data for part of an outbound crossing of the magnetosheath. The crosscorrelation analysis is carried out on data windows of $30 \mathrm{~min}$ length from 08:00 to 14:00 UT and the result of this analysis is shown in the two bottom panels of Fig. 4. The value of the cross-correlation coefficient (5th panel) and the respective deduced time lags (6th panel-crosses) are then plotted for each interval along with our calculated instantaneous time lags (dots- 6th panel). Good agreement is observed between the two sets of time lags with the maximum deviation between both values $\approx 5 \mathrm{~min}$. 


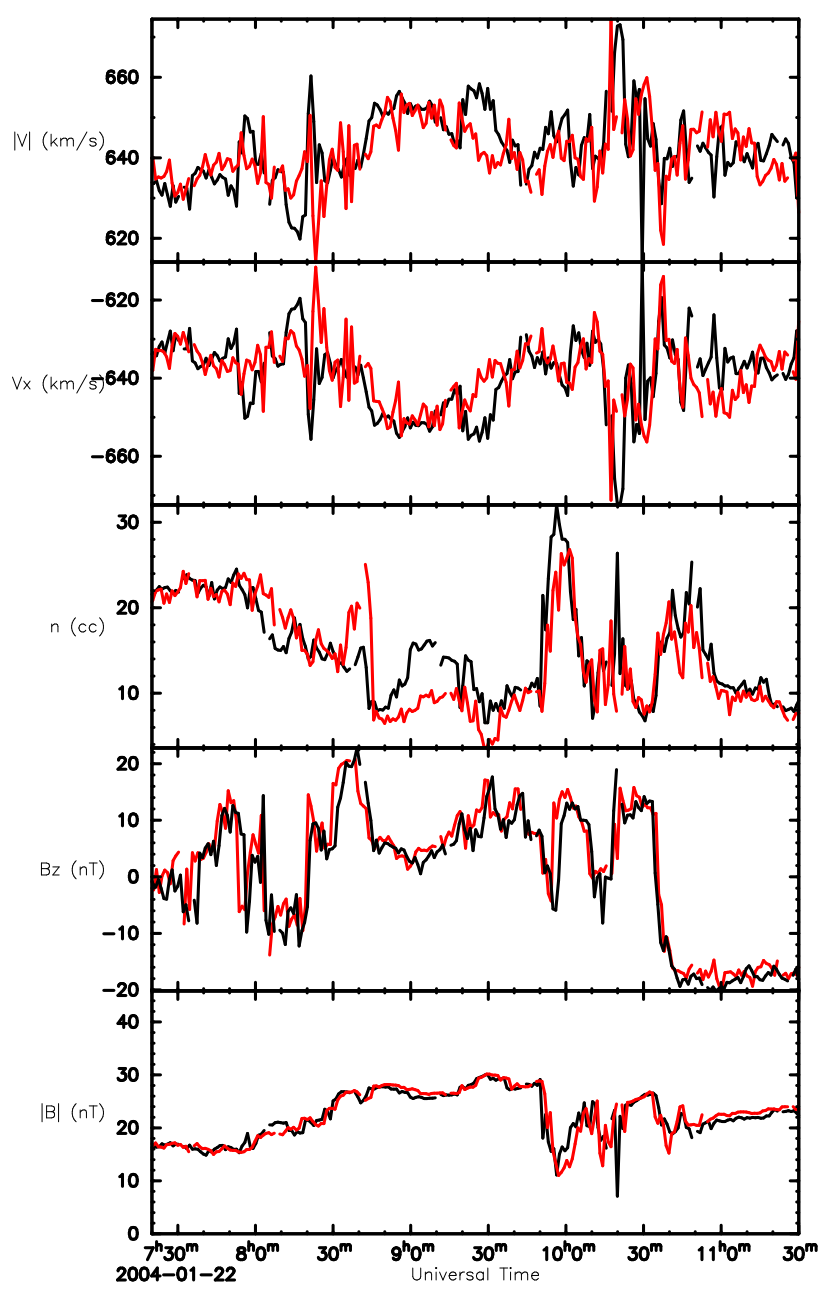

Fig. 3. Intercalibration of the Cluster and Ace data sets: A comparison of ACE solar wind (red) and Cluster 1 (black), ion velocity, ion density and magnetic field data on the 22nd of January 2004. The ACE data has been time shifted by $37 \mathrm{~min}$, corresponding to a solar wind velocity, $V_{s w} \approx 650 \mathrm{kms}^{-1}$ for illustrative purposes and shows excellent agreement with the Cluster measurements.

An additional consideration arises from our assumption regarding the orientation of the propagating solar wind planes. The normalisation assumes that the structures in the solar wind plasma propagate along the separation vector between the ACE and Cluster spacecraft. A more typical condition is arguably that plasma structure is co-aligned with a Parker spiral. For this reason we take as an example the conjunction between the ACE and Cluster spacecraft for the previous case. We then calculate the difference between the time lag for propagation of a plasma structure along the separation vector and the hypothetical time lag for a plasma structure to arrive at the Cluster spacecraft convected perpendicular to the Parker spiral angle. This is found to be of the order of $\approx 10 \mathrm{~min}$.

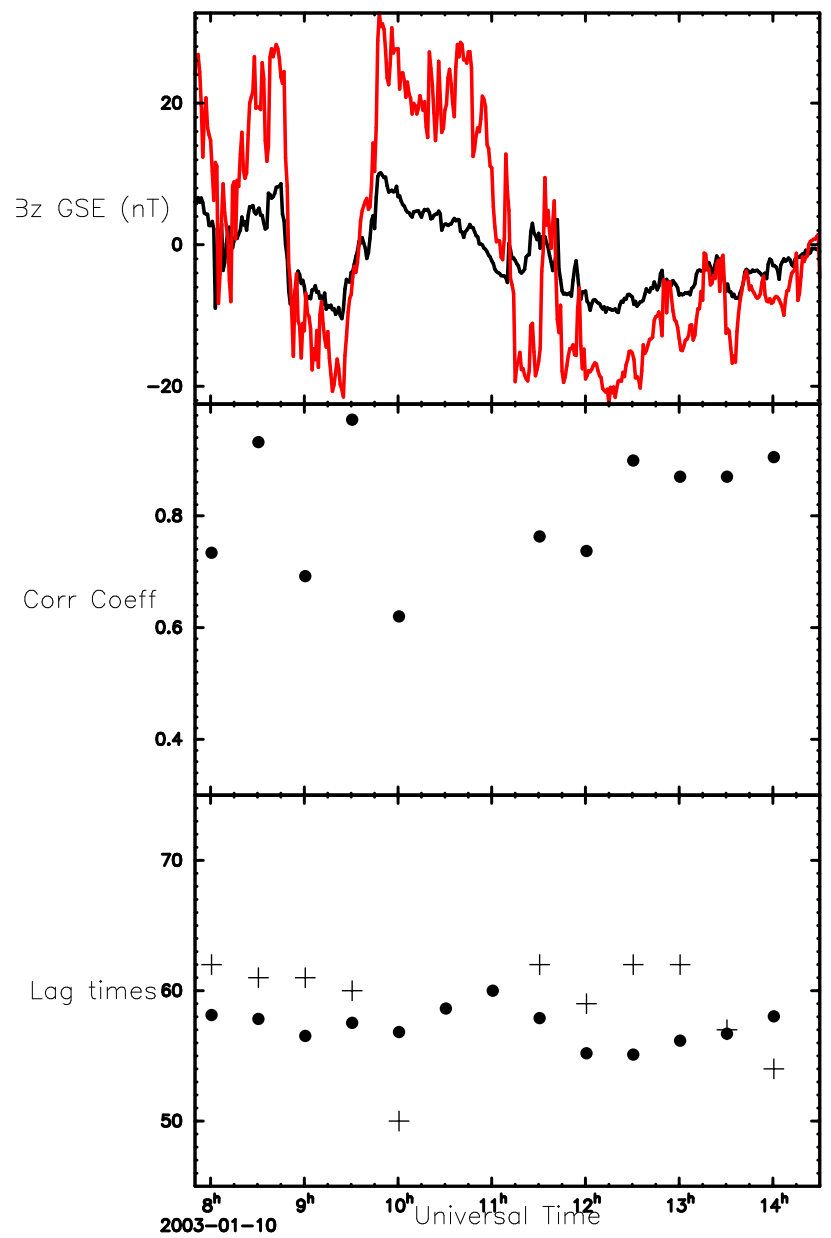

Fig. 4. Testing the lagging alogarithm: A comparison of ACE solar wind (red) and Cluster 1 (black), ion velocity, ion density and magnetic field data on the 10 of January 2003. The ACE data has been shifted by $58 \mathrm{~min}$ for illustrative purposes. Cross correlation has been carried out on the $B_{z}$ component, measured at both spacecraft (4th panel). The value of the cross correlation coefficient at intervals of $30 \mathrm{~min}$ between 08:00 and 14:00 UT is shown in panel 5. Panel 6 shows a comparison of the lag times determined from the cross correlation analysis (crosses) and the instantaneous time lagging (dots) deduced by iterating Eq. (2).

\subsection{Frame transformation}

We do not aberrate to account for the Earth's motion $\left(\approx 3^{\circ}-4^{\circ}\right)$ or any other off axis component of the solar wind velocity since these corrections have little effect on our results. In the first part of our results, Sect. 3.2, we use the GSE frame of reference. In the second part of our results, Sect. 3.3, it is necessary to transform the data into the GSM reference frame to account for the Earth's dipole variation in the $y-z$ plane. In both cases each measurement is represented by its angular location in GSE/GSM and as a fraction of radial position across the sheath. 

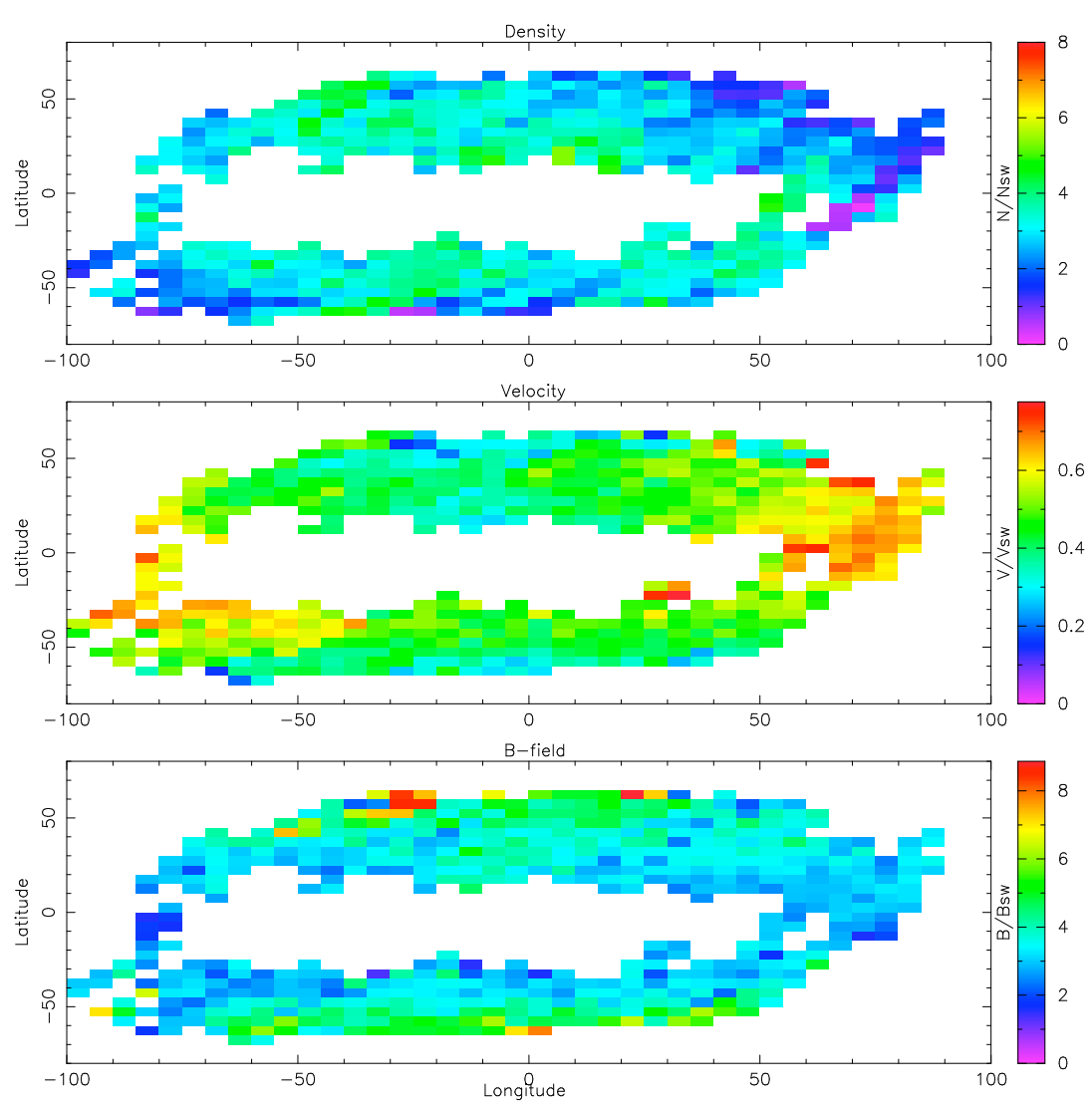

Fig. 5. Magnetosheath densities (top panel), velocities (middle panel) and magnetic field magnitude (bottom panel) measured by Cluster over the mission period January 2001-May 2004. All values have been normalised to lagged upstream values determined from ACE. Each measurement has been placed in a $5 \times 5^{\circ}$ angular bin of GSE longitude and latitude. The tiles are coloured according to their value indicated on the adjacent colour bar and represent an average over all measurements which fall into a particular 2-D geocentric angular bin.

\subsection{Data bins and data representation}

In order to illustrate our results (see Fig. 5), each measurement has been placed in a $5 \times 5^{\circ}$ angular bin of GSE longitude and latitude. Each coloured tile in these plots thus represents an average over all measurements which fall into a 2-D geocentric angular bin. The nature of the sheath coverage as discussed in Sect. 2.3 is biased by the Cluster orbit such that the most extreme northerly and southerly latitudes in this 2D representation, generally contain measurements closest to the magnetopause. As we move toward the mid-latitude region of the sheath, the region of the magnetosheath sampled is correspondingly further away from this point and closer to the bow shock.

Furthermore we compare portions of GSE longitude and latitude at different radial locations of the magnetosheath. For this purpose it is necessary to average the measurements acquired at all times over different spatial portions of the magnetosheath. In this case we use cubic bins of $0.25 \times$ magnetosheath thickness $\times 15^{\circ}$ latitude $\times 15^{\circ}$ longitude . The results are plotted in Figs. 6 and 7 where each point on a plot shows the average value and standard deviation of normalised velocity or density for each radial portion of the magnetosheath at a fixed range in latitude and longitude.

\section{Results}

\subsection{Dawn-dusk asymmetries}

Figure 5 shows the normalised sheath velocities measured by Cluster over the mission period January 2001-May 2004. The survey covers the dayside part of the magnetosheath (see Fig. 1). For the purpose of illustration the data has been binned as described in Sect. 2.8. The flow speed increases from 0.3 to 0.5 times solar wind value near the nose to up to 0.75 times the solar wind velocity at the magnetosheath flanks. The gradient of the flow towards the poles is in the opposite sense; the flow in general slowing or remaining constant as we move from equatorial latitudes to the highest and lowest latitudes in the northern and southern poles. A dawndusk asymmetry is apparent with greater velocity magnitudes measured on the dusk side of the magnetosheath in the Northern Hemisphere, whilst the converse is true of the Southern Hemisphere. The asymmetry is often greatest at the flanks of the magnetosheath and exists to a lesser extent between regions of dawn and dusk closer to noon.

In order to examine the extent of this velocity asymmetry at different regions in the magnetosheath, we compare velocity magnitudes in four paired $15^{\circ}$ longitudinal bins either 


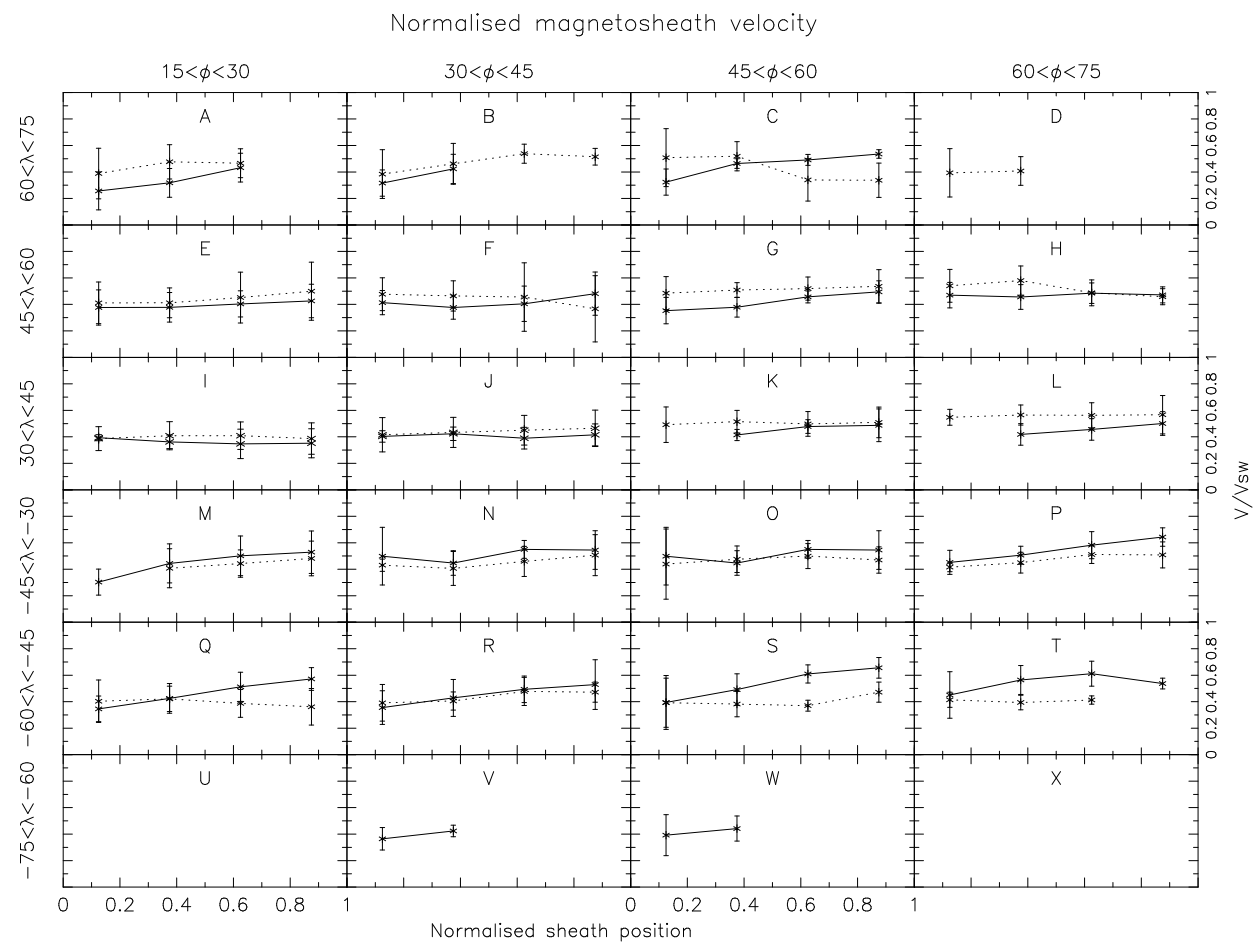

Fig. 6. The Fig. shows a matrix of panel plots of normalised velocity ratios with the absolute value of GSE longitude, $\phi$ increasing from left to right and GSE latitude, $\lambda$ decreasing from top to bottom. Each panel in the figure shows the normalised velocity for a radial cross section of the magnetosheath, where the magnetopause is at 0.0 and the bow shock at 1.0. The solid lines indicate the results for the dawn side and the dashed lines show the results for the equivalent bins at dusk. Points which are absent from these plots indicate a lack of coverage for that particular bin. The error bar is the standard deviation of the velocity estimate.

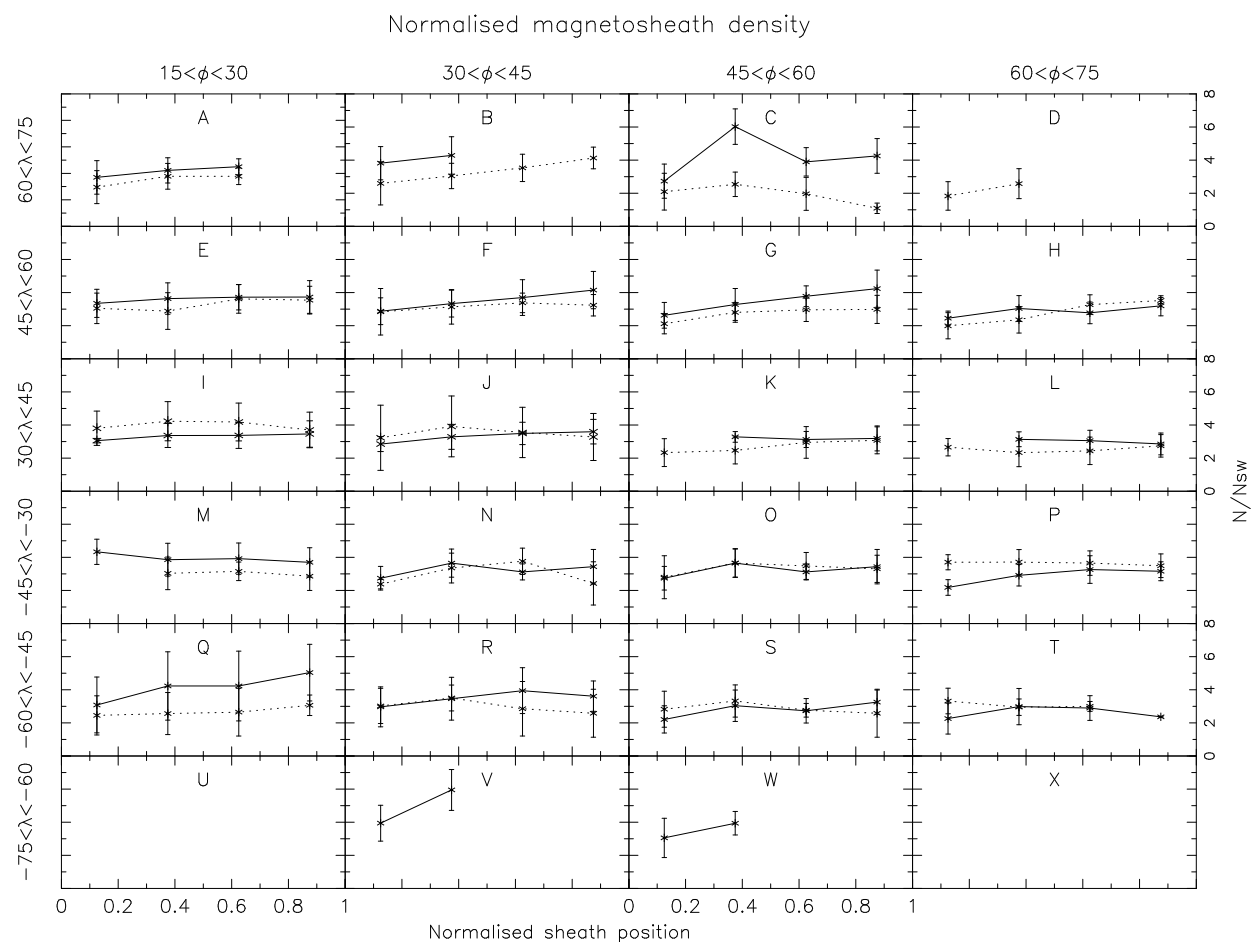

Fig. 7. Normalised density ratios presented in same format as for Fig. 6, with GSE longitude, $\phi$ increasing from left to right and GSE latitude, $\lambda$ decreasing from top to bottom. 
side of noon at 4 radial intervals between the magnetopause and the bowshock. Figure 6 shows the results of our analysis. The solid lines indicate the results for the dawn side and the dashed lines show the results for the equivalent bins at dusk. Whilst there is significant overlap of the error bars at dawn and dusk, it is clear from the plots in the figures that the velocities at dusk are higher than those at dawn in the Northern Hemisphere. If we examine the panels in Fig. 6 in closer detail, we find that differences between dawn and dusk tend to be greatest for longitudinal ranges away from noon, i.e. toward the flank and closest to the magnetopause (panels $\mathrm{A}-\mathrm{C}$ and $\mathrm{F}-\mathrm{H}$ ). In panels $\mathrm{C}$ and $\mathrm{G}$, the dawn normalised flow appears to slow and deviates from the dusk velocity as it moves towards the magnetopause. At sectors closest to noon and the ecliptic plane (panels E, I and J) there is little deviation of the dawn and dusk velocities.

Panels M to X in Fig. 6 show the results for the Southern Hemisphere. The corresponding coverage of dawn and dusk either side of noon here is poorer than in the Northern Hemisphere. However for the latitudinal and longitudinal bins where there is good coverage, the trend is that the dawn velocities are higher than those at dusk. The flow on the dawn side slows in most cases as it approaches the magnetopause, whilst there is little evidence of this occurring on the dusk side. The dawn side flow slows most dramatically from the bow shock to the magnetopause in panels M, P-T. Poor coverage of both dawn and dusk for the lowest latitudes in the Southern Hemisphere, $-75^{\circ}<\lambda<-60^{\circ}$ (see panels U$X$ in Fig. 6) means we are able to draw little conclusion on the form of the flow at dawn in this region and none at all on the form of flow at dusk.

Figure 5 shows the normalised sheath density for the survey period. Here we see some tendency for the density to decrease as we move away from the equatorial regions close to noon toward the flanks and polar latitudes. There is a difference in the values of normalised density measured for the dawn and dusk magnetosheath sectors. In the Northern Hemisphere, the density at dawn longitudes exceeds that at the equivalent dusk longitude.

To examine this in closer detail we bin the density data in an identical way to that carried out for the normalised velocity. We note that the error estimate is greater for the normalised density. Nonetheless Fig. 7 shows that the dawn densities (solid line) are higher than those at dusk (dashed line), for the Northern Hemisphere, in all panels except I and $\mathbf{J}$ which are close to noon and the equatorial plane. Away from the ecliptic plane, the density profiles, particularly for dawn, show a density minimum in the vicinity of the magnetopause.

Panels $\mathrm{M}-\mathrm{X}$ in Fig. 7 show the density results for the Southern Hemisphere. As noted before, corresponding coverage for dawn and dusk longitudinal ranges is poorer than that in the Northern Hemisphere. In this case there is no clear indication for the dusk normalised densities to exceed those at dawn. At longitudes closest to noon the dawn densities exceed those at dusk (panels M,N,Q,R), whilst furthest away at the flanks the situation appears to reverse as the dawn normalised density decreases more dramatically at the magnetopause (panels $\mathrm{P}$ and $\mathrm{T}$ ).

In Figs. 6 and 7, the majority of panels reveal dawn density and velocity minima in the high latitude sectors close to the magnetopause. This is not consistent with the gasdynamic prediction.

\subsection{Investigation of IMF control of observed assymetry}

As a next step we examine the possibility that the asymmetry might be controlled by the upstream field orientation. A cause of the magnetosheath asymmetry may be the quasi-parallel and quasi-perpendicular geometries upstream of the magnetosheath. For the typical Parker interplanetary magnetic field (IMF) direction, intersecting the Earth's bow shock at dawn this implies $\theta_{b n}<45^{\circ}$ i.e. a quasi-parallel upstream shock configuration. At dusk, the IMF is approximately tangential to the bow shock boundary with $\theta_{b n}>45^{\circ}$ producing a quasi-perpendicular upstream shock geometry. For an ortho-Parker spiral IMF configuration, the geometry of the dawn and dusk upstream shocks is reversed. The plasma conditions differ downstream of both the quasiparallel and perpendicular shocks. Transverse wave activity upstream of the quasi-parallel bow shock in particular would imply larger (if variable) tangential components of the magnetic field at the bow shock boundary and greater compression of the magnetic field downstream in the dawn magnetosheath. Since the prevailing solar wind direction corresponds to a Parker spiral orientation this could be the obvious root of downstream differences between the values of the plasma parameters at dawn and dusk. Certainly if this were the case we would anticipate the observation of a reverse of the asymmetry for a data set which contained only ortho-Parker spiral IMF.

The data is therefore subdivided into sets corresponding to Parker and ortho-Parker orientations of the upstream solar wind. To optimise the statistics available to both data sets we firstly rectifiy the data. The rectification of the magnetic field converts sectors with $+X_{G S M}$ component pointing toward the sun ("toward sectors") to sectors with a $-X_{G S M}$ component pointing away from the sun ("away sectors"). This involves flipping the $X_{G S M}, Y_{G S M}$ and $Z_{G S M}$ components of the magnetic field so that northwards IMF with positive $B_{x}$ and negative $B_{y}$ is equivalent to southwards IMF with negative $B_{x}$ and positive $B_{y}$. Likewise, southwards IMF with positive $B_{x}$ and negative $B_{y}$ is equivalent to northwards IMF with negative $B_{x}$ and positive $B_{y}$ in the rectification.

We also consider the possibility of asymmetries generated by a north-south IMF effect. The magnetosheath data for both the Parker and ortho-Parker cases are rotated into the GSM frame of reference and are subdivided according to northwards (IMF clock angle between $0^{\circ}$ and $90^{\circ}$ ), equatorial IMF ( IMF clock angle between $45^{\circ}$ and $135^{\circ}$ ) and southwards IMF (IMF clock angle between $135^{\circ}$ and $180^{\circ}$ ). These IMF cases are illustrated for the 4 radial cross-sections of the magnetosheath in the top (A-D), bottom (I-L) and middle panels $(\mathrm{E}-\mathrm{H})$ of Figs. $8 \mathrm{a}-\mathrm{d}$. The magnetosheath sector 


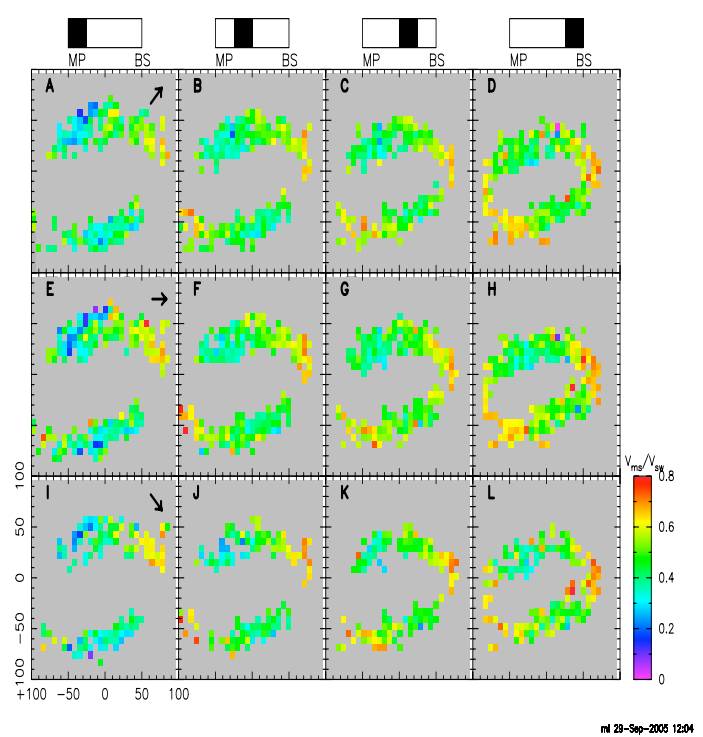

(a)

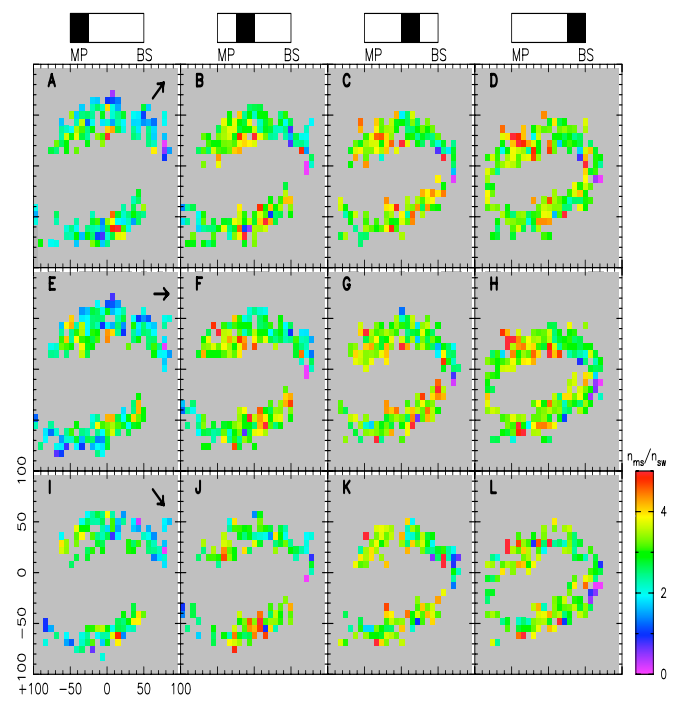

(c)

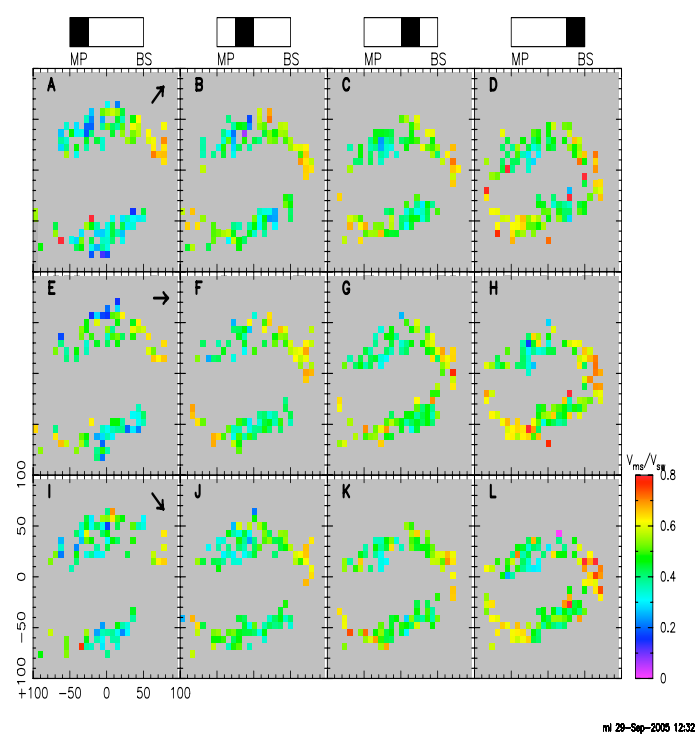

(b)

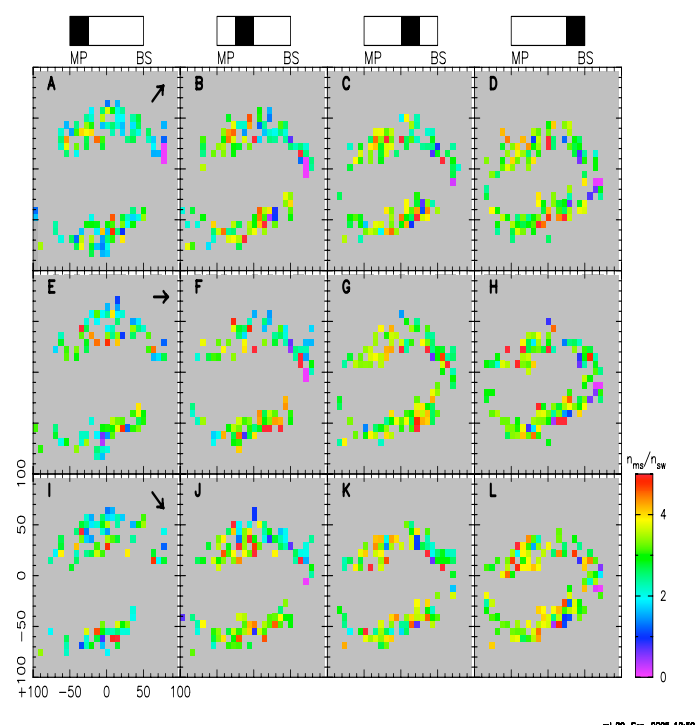

(d)

Fig. 8. Parker and ortho-Parker IMF subsets of the survey data set: Illustrated are the velocities (a) and (b) and densities (c) and (d) for the 4 radial cross-sections of the magnetosheath. The magnetosheath sector represented by a panel is indicated at the top of each of the four panel columns by a shaded box which shifts from left to right as the sector progresses outwards from the magnetopause to the bow shock boundary. The top panels $(A-D)$, bottom panels $(I-L)$ and middle panels $(E-H)$ represent northwards, southwards and equatorial IMF respectively .

represented by a panel is indicated at the top of each of the four panel columns by a shaded box which shifts from left to right as the sector progresses outwards from the magnetopause to the bow shock boundary. The upstream IMF clock angle is fixed for a row of panels and is indicated in the first panel of each row.

In the case of the data set which corresponds to Parker orientations of the upstream solar wind direction (Panels a and b of Fig. 8 ), an asymmetry is apparent in the magnetosheath velocity (see panel (a)) for all four cross sections. This asymmetry persists for northwards, southwards and equatorially aligned IMF. The velocity magnitudes are observed to reach minimum values on the dawnside of the magnetosheath close to the magnetopause. A density asymmetry exists likewise in panel (b) for all the magnetosheath cross sections and appears to be independent of the north-south component of the IMF. We note once more that the density reaches a minimum in the cross-sections closest to the magnetopause at high latitudes (panels A,E and I in Fig. 8b) as is observed in Fig. 7. Panels $\mathrm{c}$ and d of Fig. 8 correspond to the estimates of velocity and density for the four magnetosheath cross sections when the upstream IMF is ortho-Parker. Although the 


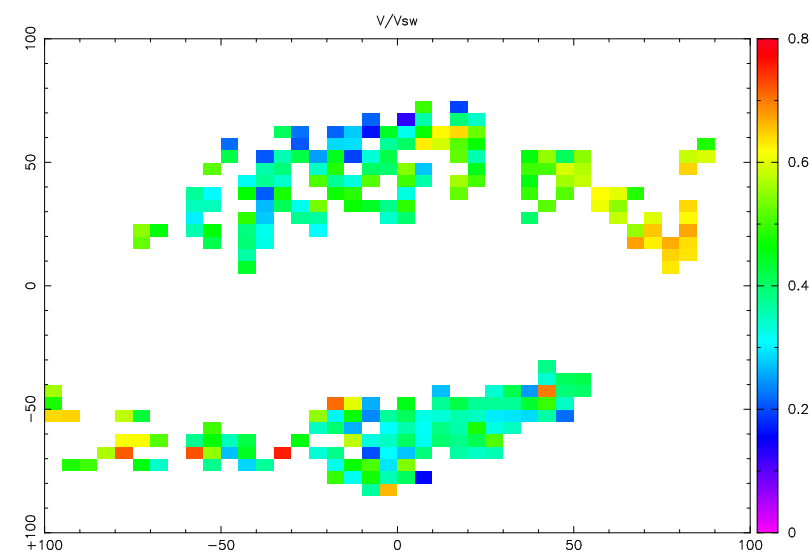

(a)

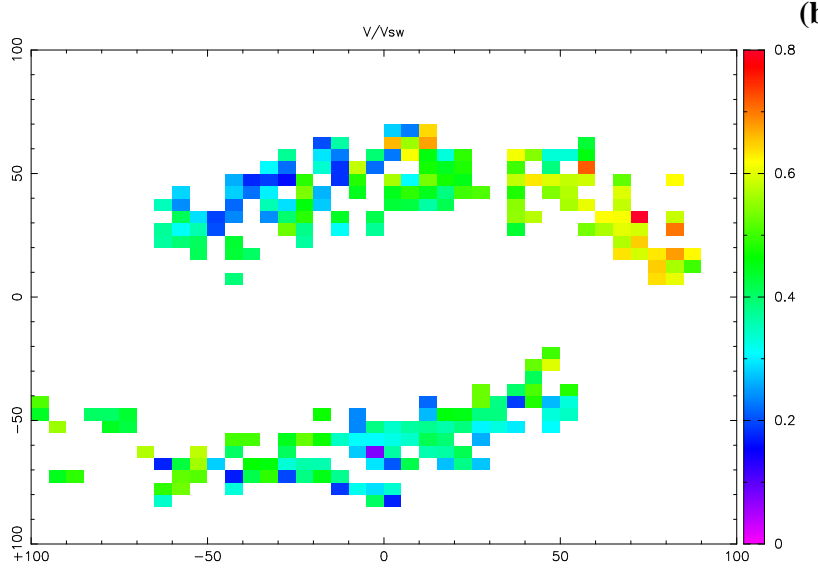

(b)

Fig. 9. Magnetosheath velocity ((a) and (b)) for IMF $B_{y}>0$ (a) and $B_{y}<0$ (b). The spectrograms shown are for the radial cross section of the magnetosheath which lies closest to the magnetopause. An examination of (a) and (b) shows that both the $B_{y}>0$ and $B_{y}<0$ data groups show an asymmetry in an identical sense

statisitcs are fewer, an asymmetry is apparent which is exactly the asymmetry observed in the Parker spiral case. This asymmetry is evident from the majority of the magnetosheath cross sections examined. A foreshock source of the asymmetry is therefore not evident from this analysis.

In the MHD description asymmetries in the magnetosheath plasma flow can be generated at cusp latitudes by the IMF $B_{y}$ component (Siscoe et al., 2002). A tangential force acts on the flow at the magnetopause boundary. This force arises from the merging of the IMF and Earth's geomagnetic field. For the pure IMF $B_{y}>0$, the force imparts a net dawnward impulse to the flow across the dayside magnetopause. The process operates exclusively at the high latitudes of the cusp region as the flow passes through the magnetopause and is therefore unlikely to be a cause of our asymmetry which is observed throughout the magnetosheath. Nonetheless, we consider whether the sign of the IMF $B_{y}$ component has an effect on the asymmetry observed for the magnetosheath plasma close to the magnetopause boundary.

We thus restrict the to the radial cross section closest to the magnetopause boundary (i.e. within $25 \%$ of the normalised distance from the magnetopause to the bow shock). The data is then grouped into ranges for which the upstream IMF $B_{y}$ GSM component is greater than or less than zero. In this way we attempt to isolate an effect on the asymmetry caused by the tangential force acting on the flow at the magnetopause boundary. It is expected that the tangential force will act in the opposite direction for $B_{y}<0$. If the $B_{y}$ direction thus exhibits an influence in our case, we might expect to observe velocity asymmetries in each of the $B_{y}>0$ and $B_{y}<0$ groups, with each asymmetry the converse of the other. However an examination of Fig. 9 shows that both the $B_{y}>0$ (Fig. 9a) and $B_{y}<0$ (Fig. 9b) data groups show an asymmetry in an identical sense.

\subsection{Sub-Alfvénic flow near the cusp}

We have calculated the Alfvén mach number, $M_{A}$ for each $5^{\circ}$ bin of latitude and longitude in identical representation to Fig. 5, with the exception that the data are know rotated into the GSM frame of reference as mentioned in Fig. 2.8. The result is illustrated in Fig. 10. The scale indicates red values for super-Alfvénic flow and blue for sub-Alfvénic. Here we see clearly that the flow at the highest and lowest latitudes is sub-Alfvénic whilst the flow tends to be super-Alfvénic at mid-latitudes and at the flanks of the magnetosheath. The dawn-dusk asymmetry observed in Sect. 3.2 is also evident here. We recall the orbital coverage bias and note that high latitude measurements lie closest to the magnetopause. The flow is thus observed to become sub-Alfvénic at the highest latitudes closest to the magnetopause.

Since flow properties near the cusps play a crucial role in reconnection, we have tabulated the results in table 1 for the bin representing the $25 \%$ of the magnetosheath which lies closest to the magnetopause. At dawn $\left(-90^{\circ}<\phi<-15^{\circ}\right)$, in the Northern Hemisphere at latitudes $60^{\circ}<\lambda<75^{\circ}$, the Alfvén mach number, $M_{A}$ is lowest and even accounting for the standard deviation lies below or close to the Alfvén speed. At lower latitudes in the dawn sector the flow is generally close to or above unity. At dusk $\left(15^{\circ}<\phi<90^{\circ}\right), M_{A}$ exceeds that on the dawn side for latitudes of $15^{\circ}<\lambda<60^{\circ}$ and the transition to sub-Alfvénic flow at the highest latitudes $\left(60^{\circ}<\lambda<75^{\circ}\right)$ is sharper. In the Southern Hemisphere the corresponding coverage of dawn and dusk latitudes is poorer and there is weaker evidence of an asymmetry for $M_{A}$ between dawn and dusk sectors. However we note that at dawn, the velocity at the most southern latitudes $\left(75^{\circ}<\lambda<60^{\circ}\right)$ is once more sub-Alfvénic.

\section{Discussion}

\subsection{The dawn-dusk asymmetry in the magnetosheath}

A striking result of our survey, which is based on four years of mid to high-latitude magnetosheath data, is the observed asymmetry between densities and velocities at dawn and dusk. Our evidence as outlined in the results section of this paper shows that the dusk velocities exceed those at dawn 
Table 1. Alfvén mach number, $M_{A}$ for the $25 \%$ of the magnetosheath adjacent to the magnetopause

\begin{tabular}{ccccc}
\hline & Northern Hemisphere & \multicolumn{3}{c}{ Southern Hemisphere } \\
Latitudes & Dawn & Dusk & Dawn & Dusk \\
\hline $15-30$ & $1.24 \pm 0.22$ & $1.61 \pm 0.23$ & - & $1.05 \pm 0.29$ \\
$30-45$ & $1.01 \pm 0.15$ & $1.78 \pm 0.58$ & $1.06 \pm 0.26$ & $1.2 \pm 0.11$ \\
$45-60$ & $1.02 \pm 0.32$ & $1.47 \pm 0.22$ & $1.29 \pm 0.27$ & $1.12 \pm 0.1$ \\
$60-75$ & $0.87 \pm 0.26$ & $0.78 \pm 0.11$ & $0.77 \pm 0.16$ & - \\
\hline
\end{tabular}

at all latitudes and longitudes in the Northern Hemisphere whilst for the majority of the dayside magnetosheath, the dawn densities are greater than those at dusk at corresponding latitudes and longitudes. The sense of this asymmetry for the normalised velocity is reversed in the Southern Hemisphere, although the result is more ambiguous for the corresponding density measurements.

We note that two previous surveys have produced a similar result. Paularena et al. (2001) showed a significant dawndusk asymmetry in Earth's magnetosheath which was found to have no dependence on IMF orientation during periods near a maximum in solar activity. Our survey (January 2001April 2004) is conducted over an era in the solar cycle which descends from solar maximum (solar maximum occurred in 2000) and is thus conducted over a period for which a large part of the data set lies closer to solar maximum than to solar minimum (with solar minimum due to occur in 2007). Likewise we have attempted to subset the data set for different orientations of both IMF $B_{y}$ and $B_{z}$ but observed no effect on the observed asymmetry. The asymmetry prevails for all orientations of the IMF.

The INTERBALL survey by Němeček et al. (2002a) revealed an excess of dawn over dusk ion flux of $\approx 20 \%$ in the magnetosheath. Again the authors examined the influence of IMF orientation, in particular IMF $B_{z}$. Whilst the dusk side was found to be more sensitive to variations in $B_{z}$ (during $B_{z}$ the ion flux was found to be enhanced at the magnetopause and bow shock), only a minor influence was found on the dawn-side. In addition the survey was carried out over a period corresponding to solar minimum (March 1995-September 1998), an interval for which Paularena et al. (2001) find only small deviations from symmetry between dawn and dusk and for which the IMF appears to be a controlling variable. The asymmetries found by the Paularena et al. (2001) and Němeček et al. (2002a) surveys corresponds to the result shown here for the Northern Hemisphere.

We note that the Interball 1 orbit in the survey of Němeček et al. (2002a) is such that the dusk magnetosheath is sampled during the Spring-early Summer season; the dawn magnetosheath during the late Autumn-Winter. The Cluster seasonal coverage mirrors this, dawn orbits occur in late WinterSpring and dusk orbits in late Autumn/mid-Winter. The IMP 8 orbit used in the Paularena et al. (2001) survey samples both dawn and dusk within a 12 day orbit. Despite the variations in seasonal sampling, the sense of the observed asym-

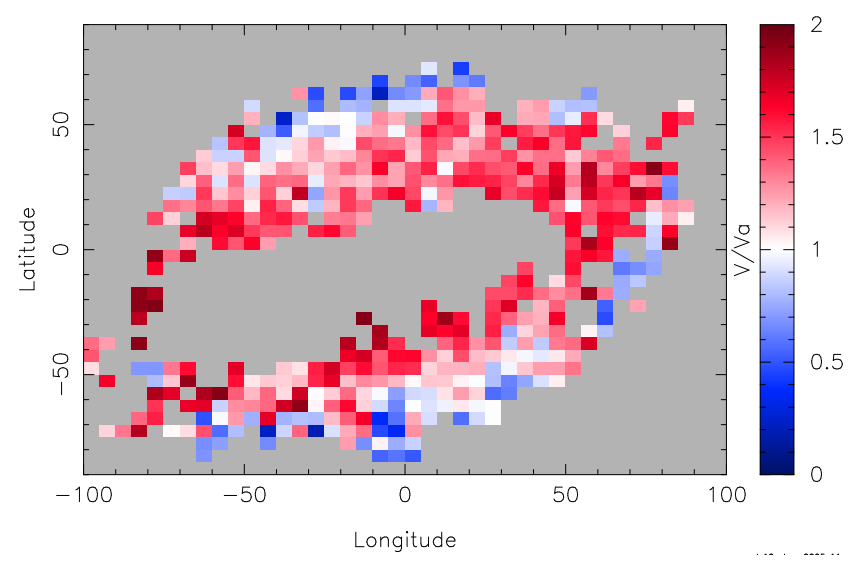

Fig. 10. Alfvén Mach numbers, $M_{A}$, measured by Cluster in the magnetosheath over the mission period January 2001-May 2004. Each measurement has been placed in a $5 \times 5^{\circ}$ angular bin of GSM longitude and latitude. The tiles are coloured according to their value indicated on the adjacent colour bar and represent an average over all measurements which fall into a particular 2-D geocentric angular bin. The scale indicates red values for super-Alfvénic flow and blue for sub-Alfvénic flow. Here we see clearly that the flow at the highest and lowest latitudes is sub-Alfvénic whilst the flow tends to be super-Alfvénic at mid-latitudes and at the flanks of the magnetosheath.

metry in the Northern Hemisphere is the same for all three surveys.

Neither the Paularena et al. (2001) nor the Němeček et al. (2002a) surveys unveil an asymmetry between the magnetosheath plasma population in the northern and Southern Hemisphere. The survey of Němeček et al. (2002a) addresses the possibility of an east-west asymmetry only. The Paularena et al. (2001) survey shows no reverse of the asymmetry between the northern and Southern Hemisphere. Both surveys sample regions of the dawn and dusk flanks which are predominantly tail-ward of the Earth $\left(-15<X_{G S E}<-20 R_{E}\right.$ and $5<X_{G S E}<-15 R_{E}$ respectively). However our survey addresses the sunward portion of the magnetosheath and this could account for the difference between our survey and that of Paularena et al. (2001).

The gas dynamic models are purely cylindrically symmetric. In the MHD description of the magnetosheath, the magnetic field directionality provides the possibility of generated asymmetries in the magnetosheath parameters. The 
Siscoe et al. (2000) MHD magnetosheath simulations examined the case of pure IMF $B_{y}>0$ (chosen as the most common direction of the IMF) and found that the flow was deflected at the high latitude magnetopause toward dawn in the Northern Hemisphere and dusk in the Southern Hemisphere. The depletion effect introduced by Zwan and Wolf (1976) which is believed to be strongest for northward IMF is also thought to favour reconnection at the highest latitudes close to the magnetopause and this in turn could explain an induced flow/density asymmetry. However since we do not observe a reversal of the Siscoe et al. (2000) asymmetry for $B_{y}>0$ nor a vanishing of the asymmetry for $B_{z}<0$ in the survey data, we conclude that the asymmetry observed throughout the magnetosheath cannot be simply due to an IMF effect operating close to the magnetopause. In our case the survey data includes data taken from the magnetosheath proper as well as the region adjacent to the magnetopause boundary and therefore does not specifically study local flux tube motion at the magnetopause boundary which is known to generate a flow asymmetry at the ionospheric level for $B_{y}>0$ (Svalgaard, 1968; Mansurov, 1969).

We have examined whether asymmetries in the plasma parameters between dawn and dusk are linked to the tendancy of these regions to lie downstream of quasi-parallel and quasi-perpendicular shock geometries for the typical Parker spiral orientation of the solar wind. Although we might expect the asymmetry to thus be reversed for the ortho-Parker spiral cases, we find that both Parker and ortho-Parker spiral IMF produce an asymmetry of the same sense.

Our result shows a dawn dusk asymmetry which predominates for almost all sectors of the magnetosheath under all IMF conditions.

\subsection{Significance of observed sub-Alfvénic-flow near the cusps}

A consequence of the slow velocity and low density observed for high latitudes close to the magnetopause is that the observed $M_{A}$ is much lower than those predicted by the gasdynamic model. In particular the flow is sub-Alfvénic at the highest latitudes, a result which is consistent with observations of Avanov et al. (2001) and Fuselier et al. (2002). It is commonly accepted that steady state reconnection can only occur in regions of sub-Alfvénic magnetosheath flow and a number of authors (Lavraud et al., 2002; Siscoe et al., 2000; Russell et al., 2000) have documented observations of reconnection occurring both equator-ward and pole-ward of the cusp region under northwards IMF. We wish to point out that the result of our statistical survey of four years of Cluster coverage in the dayside magnetosheath is that the flow does indeed become sub-Alfvénic at the high latitude magnetopause boundary in contrast to the gasdynamic model which produces super-Alfvénic flows at the same latitudes.

\subsection{Limitations}

We have examined possible sources of statistical bias which might contribute to the survey results. Firstly, the accuracy of our normalisation procedure has been checked by

1. Performing a cross-correlation analysis on data windows of 30 min length from 08:00 to 14:00 UT for a typical magnetosheath crossing. We compare the time lag deduced from cross-correlation analysis with the corresponding instantaneous time lag for the $30 \mathrm{~min}$ interval. Good agreement is observed between the two sets of time lags.

2. Calculating the uncertainty introduced by assuming propagation of plasma structure between the ACE and Cluster spacecraft. We compare the instantaneous lag with a new propagation time for plasma convected along a Parker spiral. We find that the calculated lag time exceeds our instantaneous lag time by approximately $10 \mathrm{~min}$ for plasma structure oriented tangential to the Parker spiral.

We have implicitly assumed a constant plasma velocity for both the interplanetary medium and the magnetosheath. The result of our cross correlation coefficient analysis refutes any serious implication of this for our normalisation. We therefore estimate that the accuracy of our calculated time lag is limited to within $10 \mathrm{~min}$. In any case, solar wind conditions away from interplanetary discontinuities remain constant over this period and the accuracy of normalisation is unlikely to cause a significant impact on our survey. Certainly, it could not produce a systematic error which would explain the observed asymmetry.

Nor is the asymmetry the result of systematic differences between the ion and electron moments, both of which have been used in our survey. The corrected PEACE moments are found to agree well with those from the CIS instrument when both are available (see Fig. 2).

Finally we rule out the effect of a seasonal bias due to seasonal sampling of dawn and dusk sectors by Cluster. There is indeed no obvious physical explanation, independent of the IMF, for a seasonal effect which would lead to the observed asymmetry. Moreover, the asymmetry persists when the data is cast into GSM coordinates which takes into account some of the seasonal aspects. Surveys for which there is no seasonal bias of the orbit (Paularena et al., 2001) or a seasonal bias in the opposite sense (Němeček et al., 2002a), likewise, reveal an asymmetry in the same sense as the asymmetry shown here for the Northern Hemisphere.

\section{Conclusions}

The conclusions from our survey of the dayside magnetosheath are the following.

1. The magnetosheath is observed to be non-cylindrically symmetric with regard to flow and density. Significant 
asymmetries exist between the dawn and dusk sectors and between the northern and Southern Hemisphere. In the Northern Hemisphere, the velocity is greater on the dusk side, while the density is greater on the dawn side. In the Southern Hemisphere, the situation, at least for the velocity, is reversed.

2. The dawn velocity and density profiles both show a decrease from the bow shock toward the magnetopause. Both dawn density and velocity tend to be lowest closest to the magnetopause, at polar latitudes and toward the flanks of the magnetosheath.

3. At the flanks and high latitudes in the Northern Hemisphere, the dawn flow velocity appears to decrease and deviate from the velocity at equivalent dusk longitudes as it moves towards the magnetopause. Differences between dawn and dusk in the Northern Hemisphere therefore tend to be greatest toward the flank, closest to the magnetopause.

4. Magnetosheath flow at the highest and lowest latitudes close to the magnetopause is found to be sub-Alfvénic whilst the flow tends to be super-Alfvénic at midlatitudes and at the flanks of the magnetosheath. SubAlfvénic flow is required for reconnection in the Northern Hemisphere, pole-ward of the cusp. The survey indicates that flow is indeed sub-Alfvénic in this region.

5. We observe no control of the interplanetary magnetic field (IMF) direction on the observed asymmetry and conclude there must be other as yet unknown physical processes at work. Understanding these processes remains an open but nonetheless challenging geophysical question.

Acknowledgements. We wish to thank the PEACE, CIS and FGM teams for the use of electron, ion and magnetic field data. We thank the ACE SWEPAM and MAG instrument teams and the ACE Science Center for providing the ACE data. All these sources were a vital part of this work. This work is supported by a studentship from the UK Particle Physics and Astronomy Research council.

Topical Editor T. Pulkkinen thanks P. E. Sandholt and two other referees for their help in evaluating this paper.

\section{References}

Avanov, L. A., Fuselier, S. A., and Vaisberg, O. L.: High-latitude magnetic reconnection in sub-Alfvénic flow: Interball tail observations on 29 May 1996, J. Geophys. Res., 29491-29502, doi:10.1029/2000JA000 460, 2001.

Balogh, A., Carr, C. M., Acuña, M. H., Dunlop, M. W., Beek, T. J., Brown, P., Fornaçon, K.-H., Georgescu, E., Glassmeier, K.-H., Harris, J., Musmann, G., Oddy, T., and Schwingenschuh, K.: The Cluster Magnetic Field Investigation: overview of in-flight performance and initial results, Ann. Geophys., 19, 1207-1217, 2001 ,

SRef-ID: 1432-0576/ag/2001-19-1207.

Cooling, B. M. A.: Factors influencing magnetic reconnection at the magnetopause, Queen Mary, University of London, 2003.
Cooling, B. M. A., Owen, C. J., and Schwartz, S. J.: Role of the magnetosheath flow in determining the motion of open flux tubes, J. Geophys. Res., 18763-18776, doi:10.1029/2000JA000 455, 2001.

Cowley, S. W. H. and Owen, C. J.: A simple illustrative model of open flux tube motion over the dayside magnetopause, Planet Spac., 37, 1461-1475, 1989.

Escoubet, C. P., Schmidt, R., and Goldstein, M. L.: Cluster - Science and mission overview, Space Sci. Res., 79, 1997.

Farrugia, C. J., Biernat, H. K., Erkaev, N. V., Kistler, L. M., Le, G., and Russell, C. T.: MHD model of magnetosheath flow: comparison with AMPTE/IRM observations on 24 October, 1985, Ann. Geophys., 16, 518-527, 1998 ,

SRef-ID: 1432-0576/ag/1998-16-518.

Fuselier, S. A., Waite, J. H., Avanov, L. A., Smirnov, V. M., Vaisberg, O. L., Siscoe, G., and Russell, C. T.: Characteristics of magnetosheath plasma in the vicinity of the high-altitude cusp, Planet Spac., 50, 559-566, 2002.

Génot, V. and Schwartz, S.: Spacecraft potential effects on electron moments derived from a perfect plasma detector, Ann. Geophys., 22, 2073-2080, 2004,

SRef-ID: 1432-0576/ag/2004-22-2073.

Geach, J., Schwartz, S. J., Génot, V., Moullard, O., Lahiff, A., and Fazakerley, A.: A corrector for spacecraft calculated electron moments, Ann. Geophys., 23, 931-943, 2005,

SRef-ID: 1432-0576/ag/2005-23-931.

Johnstone, A. D., Alsop, C., Burge, S., Carter, P. J., Coates, A. J., Coker, A. J., Fazakerley, A. N., Grande, M., Gowen, R. A., Gurgiolo, C., Hancock, B. K., Narheim, B., Preece, A., Sheather, P. H., Winningham, J. D., and Woodliffe, R. D.: Peace: a Plasma Electron and Current Experiment, Space Sci. Res., 79, 351-398, 1997.

Lavraud, B., Dunlop, M. W., Phan, T. D., Rème, H., Bosqued, J.-M., Dandouras, I., Sauvaud, J.-A., Lundin, R., Taylor, M. G. G. T., Cargill, P. J., Mazelle, C., Escoubet, C. P., Carlson, C. W., McFadden, J. P., Parks, G. K., Moebius, E., Kistler, L. M., Bavassano-Cattaneo, M.-B., Korth, A., Klecker, B., and Balogh, A.: Cluster observations of the exterior cusp and its surrounding boundaries under northward IMF, Geophys. Res. Lett., 29, 56, doi:10.1029/2002GL015 464, 2002.

Mansurov, S.: New evidence of a relationship between magnetic fields in space and on earth, Geomagn. Aeron., 9, 622, 1969.

Němeček, Z., Šafránková, J., Zastenker, G. N., Pišoft, P., Paularena, K. I., and Richardson, J. D.: Observations of the radial magnetosheath profile and comparison with gasdynamic model predictions, Geophys. Res. Lett., 27, 2801-2804, doi:10.1029/2000GL000 063, 2000.

Němeček, Z., Šafránková, J., Zastenker, G. N., Pišoft, P., and Jelínek, K.: Low-frequency variations of the ion flux in the magnetosheath, Planet Spac., 50, 567-575, 2002a.

Němeček, Z., Šafránková, J., Zastenker, G. N., Pišoft, P., and Paularena, K. I.: Spatial distribution of the magnetosheath ion flux, Adv. Space Res., 30, 2751-2756, 2002b.

Paschmann, G., Baumjohann, W., Sckopke, N., Papamastorakis, I., and Carlson, C. W.: The magnetopause for large magnetic shear - AMPTE/IRM observations, J. Geophys. Res., 91, 11099 $11115,1986$.

Paschmann, G., Baumjohann, W., Sckopke, N., Phan, T.-D., and Luehr, H.: Structure of the dayside magnetopause for low magnetic shear, J. Geophys. Res., 98, 13 409-13 423, 1993.

Paularena, K. I., Richardson, J. D., Kolpak, M. A., Jackson, C. R., and Siscoe, G. L.: A dawn-dusk density asymmetry 
in Earth's magnetosheath, J. Geophys. Res., 25 377-25394, doi:10.1029/2000JA000 177, 2001.

Peredo, M., Slavin, J. A., Mazur, E., and Curtis, S. A.: Threedimensional position and shape of the bow shock and their variation with Alfvenic, sonic and magnetosonic Mach numbers and interplanetary magnetic field orientation, J. Geophys. Res., 100, 7907-7916, 1995.

Phan, T.-D., Paschmann, G., Baumjohann, W., Sckopke, N., and Luehr, H.: The magnetosheath region adjacent to the dayside magnetopause: AMPTE/IRM observations, J. Geophys. Res., 99, 121-141, 1994.

Rème, H., Aoustin, C., Bosqued, J. M., Dandouras, I., Lavraud, B., Sauvaud, J. A., Barthe, A., Bouyssou, J., Camus, T., Coeur-Joly, O., Cros, A., Cuvilo, J., Ducay, F., Garbarowitz, Y., Medale, J. L., Penou, E., Perrier, H., Romefort, D., Rouzaud, J., Vallat, C., Alcaydé, D., Jacquey, C., Mazelle, C., D’Uston, C., Möbius, E., Kistler, L. M., Crocker, K., Granoff, M., Mouikis, C., Popecki, M., Vosbury, M., Klecker, B., Hovestadt, D., Kucharek, H., Kuenneth, E., Paschmann, G., Scholer, M., Sckopke, N., Seidenschwang, E., Carlson, C. W., Curtis, D. W., Ingraham, C., Lin, R. P., McFadden, J. P., Parks, G. K., Phan, T., Formisano, V., Amata, E., Bavassano-Cattaneo, M. B., Baldetti, P., Bruno, R., Chionchio, G., di Lellis, A., Marcucci, M. F., Pallocchia, G., Korth, A., Daly, P. W., Graeve, B., Rosenbauer, H., Vasyliunas, V., McCarthy, M., Wilber, M., Eliasson, L., Lundin, R., Olsen, S., Shelley, E. G., Fuselier, S., Ghielmetti, A. G., Lennartsson, W., Escoubet, C. P., Balsiger, H., Friedel, R., Cao, J.-B., Kovrazhkin, R. A., Papamastorakis, I., Pellat, R., Scudder, J., and Sonnerup, B.: First multispacecraft ion measurements in and near the Earth's magnetosphere with the identical Cluster ion spectrometry (CIS) experiment, Ann. Geophys., 19, 1303-1354, 2001,

SRef-ID: 1432-0576/ag/2001-19-1303.

Roelof, E. C. and Sibeck, D. G.: Magnetopause shape as a bivariate function of interplanetary magnetic field $B_{z}$ and solar wind dynamic pressure, J. Geophys. Res., 98, 21 421, 1993.

Russell, C. T., Le, G., and Petrinec, S. M.: Cusp observations of high-and low-latitude reconnection for northward IMF: An alternative view, J. Geophys. Res., 5489-5496, doi:10.1029/1999JA900 489, 2000.
Šafránková, J., Hayosh, M., Němeček, Z., and Přech, L.: Multiscale processes in the Earth's Magnetosphere: From Interball to Cluster, chap. Magnetosheath Investigations: Interball contribution to the topic, 73-94, Kluwer Academic Publishers, 2004.

Shue, J.-H., Song, P., Russell, C. T., Chao, J. K., and Yang, Y.H.: Toward predicting the position of the magnetopause within geosynchronous orbit, J. Geophys. Res., 105, 2641-2656, 2000.

Siscoe, G. L., Erickson, G. M., Sonnerup, B. U. Ö., Maynard, N. C., Siebert, K. D., Weimer, D. R., and White, W. W.: Deflected magnetosheath flow at the high-latitude magnetopause, J. Geophys. Res., 12 851-12 858, doi:10.1029/1999JA000 268, 2000.

Siscoe, G. L., Crooker, N. U., Erickson, G. M., Sonnerup, B. U. Ö., Maynard, N. C., Schoendorf, J. A., Siebert, K. D., Weimer, D. R., White, W. W., and Wilson, G. R.: MHD properties of magnetosheath flow, Planet Spac., 50, 461-471, 2002.

Song, P., Russell, C. T., and Thomsen, M. F.: Slow mode transition in the frontside magnetosheath, J. Geophys. Res., 97, 82958305, 1992a.

Song, P., Russell, C. T., and Thomsen, M. F.: Waves in the inner magnetosheath - A case study, Geophys. Res. Lett., 19, 21912194, 1992b.

Southwood, D. J. and Kivelson, M. G.: Magnetosheath flow near the subsolar magnetopause: Zwan-Wolf and SouthwoodKivelson theories reconciled, Geophys. Res. Lett., 22, 32753278, doi:10.1029/95GL03 131, 1995.

Spreiter, J. R. and Stahara, S. S.: A new predictive model for determining solar wind-terrestrial planet interactions, J. Geophys. Res., 85, 6769-6777, 1980.

Spreiter, J. R., Summers, A. L., and Alksne, A. Y.: Hydromagnetic flow around the magnetosphere, Planet Spac., 14, 223-253, 1966.

Svalgaard, L.: Sector structure of the interplanetary magnetic field and daily variation of the geomagnetic field at high latitudes, chap. R-6, Geophys. Pap., Danish Meteorol. Inst., Carlottenlund, 1968.

Zwan, B. J. and Wolf, R. A.: Depletion of solar wind plasma near a planetary boundary, J. Geophys. Res., 81, 1636-1648, 1976. 